\title{
Predictors of returning for second round screening at a population based mammographic screening programme in Melbourne, Australia
}

\author{
Jill Cockburn, Penelope Schofield, Victoria White, David Hill, Ian Russell
}

\begin{abstract}
Objective - To examine factors associated with returning for second round mammography screening.

Setting - This was a population based mammographic screening programme in Melbourne, Australia.

Design - A cohort design was used whereby 668 women were interviewed before the screening programme began and attendance for both first and second round screening was ascertained from programme records. The cohort sample was drawn from two regions of a defined area (close to and distant from the screening centre).

Main results - Of the 315 women who attended for first round screening, $75 \%$ from the proximal sample and $70 \%$ from the distal sample returned for second round screening. Attendance at the second round was predicted by the following: the method of recruitment for first round screening, with women who required a letter of invitation and a reminder being less likely to reattend than those who initially attended in response to a community campaign $(\mathrm{OR}=0.34$; CI 0.19,0.61); mammographic history before the initial screen, with women who reported previous diagnostic mammography being more likely to reattend than those who did not $(O R=2.97$; CI 1.01,8.9); stated intention of attending for the initial screen, with those with weakest intention of attending for their first round being less likely to attend for second round $(O R=0.44$; $C I$ $0.23,0.85)$; and increasing scores on a scale that measured preventive orientation to health $(O R=1.24 ;$ CI 1.02,1.50).

Conclusion - Our findings corroborate other findings of reduced long term attendance for screening from people who are "reluctant participants" initially.
\end{abstract}

( $\mathcal{f}$ Epidemiol Community Health 1997;51:62-66)

Sufficient regular attendance among women in the target population will be a critical determinant of the success of population based mammographic screening programmes for reducing population mortality from breast cancer. In Australia, calculations concerning the benefit of mammography are based on the premise that $70 \%$ of women in the group targeted by the National Program for the Early Detection of Breast Cancer will have regular screening mammograms at two yearly intervals. To ensure that the women recruited for initial screening continue to participate with regular screening in the programme is therefore a challenge.

In contrast to the large body of research on initial attendance, ${ }^{1}$ comparatively little research has been conducted on factors associated with returning regularly for screening, ${ }^{2-8}$ despite reports that participation rates tend to fall off in successive screening rounds. ${ }^{28-10}$ An understanding of the factors that predict reattendance at screening is essential so that strategies for encouraging participation can be effectively targeted.

We have previously reported on the factors which predict attendance at first round screening among a cohort of women originally interviewed before the beginning of a pilot mammographic screening programme in Victoria. ${ }^{11}$ We are now able to examine the patterns of attendance in the programme for second round screening among the women who attended initially, and to examine the factors that predict reattendance.

\section{Setting}

The National Program for the Early Detection of Breast Cancer offers a free mammographic screening service to women aged over 40 at accredited screening and assessment centres throughout Australia. Although women over 40 are eligible for free mammography, only women aged 50 to 69 are actively recruited to the programme. Before the programme had been established nationally, a number of pilot programmes were undertaken around Australia. The pilot programme in metropolitan Melbourne, Victoria began in 1988 and, at the end of its pilot phase, became part of the national programme. The target population for the pilot programme was defined as women aged 50 to 69 living in any of 34 postcodes surrounding the programme centre. This comprised an area of approximately $905 \mathrm{~km}^{2}$, with 475000 residents and 39404 women aged 50 to 69. Compared with the Australian population as a whole, a larger proportion was born overseas $(36 \% \mathrm{v} 22 \%)$. The most frequently reported countries of birth were Italy $(6 \%)$, UK (3\%), and Greece (3\%).

\section{Participants and methods}

The study methods have been described in detail elsewhere. ${ }^{112}$ Briefly, before the pilot 
Table 1 Characteristics of the women from the proximal and distal samples who returned for the second round of mammograpghy screening

\begin{tabular}{llccc}
\hline & & $\begin{array}{c}\text { Proximal } \\
(n=167) \\
\%\end{array}$ & $\begin{array}{l}\text { Distal } \\
(n=148) \\
\%\end{array}$ & $\begin{array}{c}\text { p value } \\
\text { for } \chi^{2}\end{array}$ \\
\hline Age(y) & $50-59$ & 42.5 & 70.3 & \\
Home tenure: & $60-69$ & 57.5 & 29.7 & $<0.0001$ \\
& Renting & 5.5 & 6.8 & \\
& Buying & 4.3 & 43.2 & \\
Language spoken & Own & 87.1 & 45.9 & $<0.0001$ \\
at home: & Ener & 3.1 & 4.1 & \\
Education: & Other language & 19.0 & 12.8 & \\
& Lower secondary or less & 81.0 & 87.2 & 0.26 \\
& Upper secondary or trade & 44.4 & 56.5 & \\
Employment status: & Post secondary diploma & 8.0 & 35.4 & \\
& Employed & 43.8 & 56.2 & 0.25 \\
& & & & 0.05 \\
\hline
\end{tabular}

screening programme began, 668 women from the target population (women aged between 50 and 69 who lived in a defined geographical area around the programme) were interviewed in their homes. The sample size was intended to detect a $12 \%$ difference on measured variables between attenders and non attenders with alpha $=0.05$ and beta $=0.80$. Electoral lists were used to draw two separate random samples for our survey - one living within a $2 \mathrm{~km}$ radius of the programme (the "proximal" sample), the other from an area $10-20 \mathrm{~km}$ away (the "distal" sample). This was done to control for the effects of distance from the programme in the analysis. The interview covered nine topic areas that it has been suggested might influence decisions about attendance at the screening programme. These topic areas were as follows: experience with breast disease and prior mammography; perceived susceptibility to and concern about breast cancer; knowledge about breast cancer and screening; benefits and barriers associated with mammography; participation in other preventive health behaviours; social influence; ability to get to the programme; health related character traits; and sociodemographic characteristics.

About two months after the women had been interviewed, the pilot screening programme opened. Recruitment for first round screening, conducted over two years, was achieved by a public campaign (based on local newspaper articles and promotion of the programme to the community) followed by personal letters of invitation and reminders to women who did not respond to the community campaign. Three hundred and fifteen of the women in our sample (167 from of the proximal sample and 148 from the distal sample) attended the screening programme. The public recruitment campaign promoting the service continued after the pilot programme became part of the national programme, with women who attended for their first screen in the pilot programme also being invited by letter for rescreening two years later. The reattendance data of women from our sample was ascertained from programme records, along with information on the clinical outcome of the first screening visit and the time taken to notify women of their results. None of the women in our sample was found to have breast cancer at the first screen. The sample size of 315 first round attenders is sufficient to detect a difference of $17 \%$ on measured variables between second round attenders and non-attenders.

ETHICS

The study was approved by the Ethics Committee of the Anti-Cancer Council of Victoria.

\section{Results}

SAMPLE

The demographic profiles of the women from the proximal and distal samples who returned for second round screening are shown in table 1. The women in the proximal sample were significantly older $\left(\chi^{2}=24.28 ; \mathrm{df}=1 ; \mathrm{p}<0.0001\right)$, more likely to own their own home $\left(\chi^{2}=71.43\right.$; $\mathrm{df}=3 ; \mathrm{p}<0.0001$ ), and less likely to be employed $\left(\chi^{2}=3.84 ; \mathrm{df}=1 ; \mathrm{p}=0.05\right)$ than women in the distal sample.

\section{ATTENDANCE AT SECOND ROUND}

There was no significant difference in the reattendance rates for women in the proximal $(75 \%)$ and distal samples $(70 \%) ; \chi^{2}=0.83$; $\mathrm{df}=1 ; \mathrm{p}=0.36$ ). The median time from first to second screen was 27 months.

PREDICTORS OF ATTENDANCE AT SECOND ROUND Logistic regression was used to predict attendance at the second screening round. Each measured variable was independently tested for association with the outcome, after controlling for the effects of region and, hence, the possible confounding effects of the different demographic profiles. Table 2 displays all variables with associations with attendance of $p<0.05$.

\section{Experience with breast disease and mammography}

Women who reported at the interview that they had previously undergone a diagnostic mammogram were more likely to return for second round screening in the mammography screening programme than women who had not. A family history of breast cancer, having had a breast lump, and the amount of previous contact with people with breast cancer were not significantly associated with reattendance.

\section{Perceived susceptibility to and concern about breast cancer}

Perceptions of susceptibility to breast cancer and the intensity of concern about breast cancer measured before the beginning of the pilot screening programme were not associated with attendance at second round screening.

\section{Knowledge}

Knowledge of risks for breast cancer and of available treatments for early detected breast cancer were measured at the interview. Knowledge of these issues at this time was not associated with attendance at second round screening. 
Table 2 Significant predictors of attendance for the second round of the mammographic screening programme

\begin{tabular}{|c|c|c|c|c|c|}
\hline Variable & $\%$ returned & $\chi^{2} p$ value $e^{*}$ & $d f$ & $O R^{*}$ & $95 \% C I$ \\
\hline Mammography prior to initial screen:đ & & 0.05 & 2 & & \\
\hline None $(n=257)$ & 72 & & & $1.00 t$ & \\
\hline Diagnostic $(n=34)$ & 87 & & & 2.97 & $1.01,8.9$ \\
\hline Screening $(n=22)$ & 64 & & & 0.68 & $0.27,1.7$ \\
\hline Score on preventative orientation scale:- & & $0.03 \ddagger$ & $1^{3}$ & $1.24 \Omega$ & $1.02,1.50$ \\
\hline Lowest quintile $(n=46)$ & 61 & & & & \\
\hline 2nd quintile $(n=55)$ & 71 & & & & \\
\hline 3rd quintile $(n=44)$ & 68 & & & & \\
\hline 4th quintile $(n=63)$ & 78 & & & & \\
\hline Highest quintile $(n=64)$ & 80 & & & & \\
\hline Stated intention of attending for initial screen:ी & & 0.002 & 2 & & \\
\hline Likely to attend $(n=235)^{\circ}$ & 77 & & & $1.00 t$ & \\
\hline Neither likely nor unlikely $(n=31)$ & 61 & & & 0.47 & $0.22,1.04$ \\
\hline Unlikely to attend $(n=49)$ & 60 & & & 0.44 & $0.23,0.85$ \\
\hline Method of recruitment initial screen:ף & & 0.0008 & 2 & & \\
\hline Public campaign only $(n=156)$ & 80 & & & $1.00+$ & \\
\hline Public campaign \& personalised invitation $(n=53)$ & 77 & & & 0.84 & $0.39,1.79$ \\
\hline Public campaign \& invitation \& reminder $(n=101)$ & 58 & & & 0.34 & $0.19,0.61$ \\
\hline
\end{tabular}

* Adjusted for region.

† Reference category.

$¥ \chi^{2}$ trend (adjusted for region)

$\oint$ OR associated with one unit increase in derived score (eg, moving from lowest to next highest quintile).

aी Some missing data.

Perceptions of benefits and barriers associated with mammography

Perceptions of expected embarrassment, radiation risk, and accuracy of mammography measured at the interview were not associated with attendance at second round screening.

Other preventive health behaviours

An up to date Pap test screening history and frequency of breast self examination were not significantly related to reattendance.

Social influence

Social influence on the respondent was measured for five referent groups: friends, her partner, children, co-workers, and her doctor. None of these perceived influences was significantly associated with reattendance.

\section{Access issues}

A number of measures of access to the screening service were obtained. These included actual distance to the programme, perceived difficulty of getting to the programme, familiarity with the areas around the screening service, and work and home commitments. None of these factors was associated with attendance for second round screening.

\section{Health related character traits}

Increasing scores on a scale that measured preventive orientation toward health were associated with re-attendance (table 2). This scale contained three items asking for agreement on a five point Likert scale. The items in the scale were as follows:

1 It is a good idea to have medical check ups; 2 Having a check up would make me feel more confident about my health; and

3 Having medical exams and physical check ups just seems like too much trouble.

The scoring of the last item was reversed before scale computation. No association was found between second round attendance and health locus of control, dispositional optimism, medical anxiety, beliefs about the priority of health, and being tied to a routine.

\section{Sociodemographic characteristics}

Age at interview, home ownership, employment status, speaking a language other than English, and club membership were not associated with returning for second round screening.

Intention to attend for first round screening

Women who stated the strongest intentions about attending for first round screening were significantly more likely to return for second round screening also (see table 2 ).

\section{Method of recruitment for first round screening} Compared with women who attended for an initial screen in response to the community campaign before receiving a letter of invitation, women who did not attend till they had received both a written personal invitation and a reminder letter were less likely to return for their second round screen (table 2).

\section{Outcome of first visit}

There was no significant association between second round attendance and having been recalled for further assessment at the initial visit, either because of a suspected abnormality or because of a technical problem in the mammogram, and returning for second round screening. The time between first visit and notification of results from that visit also did not predict attendance at the next round of screening.

\section{Discussion}

In this study, the strongest predictor of attendance at second round screening in the programme was the method of recruitment for first round screening. Women who required the most intensive recruitment efforts to attend for 
the initial screening were those least likely to return. In line with this, women who initially indicated that they did not intend to have a screening mammogram at the programme were least likely to return for second round screening. This finding corroborates an early finding from the original HIP study of reduced long term participation from what Fink et al termed "the reluctant participants" at initial screen, and confirms the adage "past behaviour is the best predictor of future behaviour". ${ }^{13}$

Fink et al cautioned that the diminishing participation of respondents in subsequent screenings should not be taken to indicate that more intensive efforts to recruit women into a screening programme are not worth the effort. The results of our earlier study support this, as the letter of invitation and the reminder contributed significantly to initial attendance rates. As reported in our earlier study, the invitation boosted attendance at first round screening in the proximal sample from $35 \%$ attending in response to the public campaign and before being sent the letter, to $58 \%$ following the reminder letter; and in the distal sample from $17 \%$ attending before the letters to $46 \%$ attending after the second reminder letter. ${ }^{11}$ Moreover, as with the study of Fink et $a l$, we found that although the level of participation for second round screening was not as high for those who had needed more intensive recruitment efforts at the first round, $77 \%$ of those who received a personal letter and $58 \%$ of those who required both a letter and a reminder attended for second round screening.

The other variables that differentiated those women who returned for subsequent round screening in the programme from those who did not were similar to some found in other studies. Fink et $a l^{2}$ found that the only significant relationship between completed screening examinations and attitudes toward screening was in response to an item, "physical examinations just make you worry; its like looking for trouble". Women who disagreed with this item were more likely to complete the screening. This is similar to our finding of a significant trend towards higher scores on a preventive orientation scale being associated with subsequent attendance.

A history of mammograms has also been found in other studies to determine regular attendance at a screening programme. ${ }^{35}$ Surprisingly, in our study, compared with women who had never had a mammogram, those who had had a diagnostic mammogram before their initial attendance in the screening programme were more likely to return for second round screening, while women who had had a previous screening mammogram were not. Given that the pilot screening programme in our study was the first of its kind in Victoria, screening mammograms before the initial visit must have been obtained at private services outside the organised programme. It is possible that women returned to this service for further mammograms rather than continuing with the national programme. If it is the case that, rather than stopping having screening mammograms at all, women in our sample were choosing to have screening mammograms outside the national programme, this may explain why many of our hypothesised variables, particularly those related to perceptions of susceptibility, were not related to second round attendance in the programme. However, this is speculative as we do not know which, if any, women were having subsequent mammograms outside the national programme.

Nor do we have any information of women's perceptions of their initial visit at the screening programme. This is likely to be an important determinant for shaping women's decisions about reattendance at the programme. This lack of information, along with the long time period between measurement of variables and the behaviour of interest, may explain why the variables assessing knowledge and the perceptions of benefits and barriers of the screening process did not differentiate between attenders and non-attenders at second round screening. It seems reasonable to expect that intervening events, such as promotional campaigns and, most notably, the screening visit itself, would have affected knowledge and perceptions of embarrassment, accuracy, and radiation risk thereby attenuating any relationships between these variables and the target behaviour. This is supported by studies from other programmes that show a difference in knowledge and attitudes as a result of campaigns to promote mammographic screening. ${ }^{14}$

A number of the variables that predicted attendance at first round screening did not predict attendance at second round screening - for example, a perception of being at least at some risk for breast cancer, good knowledge about treatments for breast cancer, and lack of concern about radiation. ${ }^{11}$ Apart from the effect of the screening visit and other intervening events, other explanations are possible for this. The analysis reported in this paper only includes those women who attended for first round screening. Therefore, we are looking at a homogeneous sample of women on the variables measured. There may have been insufficient variance on these variables to detect change. Our study also had low power to detect small differences. It is also possible that, in fact, factors that are important for influencing first round screening are not those that are important for second round screening. If this is the case, promotion for second round screening would not need to focus on all the factors used to promote first round screening. Further investigation of this finding is needed in larger studies that also include a measure of acceptability of the initial screening visit.

In our sample, around $70 \%$ of those attending for first round screening subsequently attended for second round screening in the programme. However, as only $51 \%$ of our sample attended for the initial round of screening, ${ }^{11}$ the second round attendance represents only $36 \%$ of those initially interviewed. If similar rates for rescreening are occurring at other centres, and in the national screening programme as a whole, this is a matter of concern as it is clearly below the $70 \%$ regular participation rate required for a significant re- 
duction in population mortality from breast cancer. Data on rates of second round screening in the national programme in Australia are not yet available. It is possible that some women may be having screening mammograms at services that are not part of the national programme. It could be argued that it is in a woman's best interests to have a screening mammogram as part of the national programme where accreditation ensures a high quality screening service. It can not be assumed that screening received at non-programme services is of the same quality as that received in the programme. Further research is needed to identify factors influencing decisions about rescreening in the programme. The information collected should include perceptions of the experience at first round screening and data should be collected close to the time of scheduled rescreening so that identified factors will be those most likely to be influencing decisions at that time.

We would like to thank Li-chun Quang and Susy Alessandri for retrieving data from breast screen records; Marco Cappiello analysis and the women who participated in the interviews.

The research was funded by the Anti-Cancer Council of The research was funded by the Anti-Cancer Council of Cockburn is supported by a grant from the Victorian Health Cockburn is supported

1 Vernon SW, Laville EA, Jackson GL. Participation in breast screening programs: A review. Soc Sci Med 1990;30:1107118 .
2 Fink R, Shapiro S, Roester M. Impact of efforts to increase participation in repetitive screenings for early breast cance detection. Aust $\mathcal{F}$ Public Health 1972;?:328-36.

3 Lerman C, Trock B, Rimer BK, Boyce A, Jepson C, Engstrom PF. Psychological and behavioural implications of abnormal mammograms. Ann Intern Med 1991;114 657-61.

4 Lerman C, Rimer B, Trock B, Balshem A, Engstrom PF Factors associated with repeat adherence to breast cancer screening. Prev Med 1990;19:279-90.

5 Marshall G. A comparative study of re-attenders and nonre-attenders for second triennial national breast cancer screening program appointments. 7 Public Health Med 1994;16:79-86.

6 Orton M, Fitzpatrick R, Fuller A, Mant D, Milynek, Thorogood $M$. Factors affecting women's response to an invitation to attend for a second breast cancer screening invitation to attend for a second breast cancer

7 Meldrum P, Tumbull D, Dobson HM, Colquhuoun C, Gilmour WH, McIlwaine GM. Tailored written invitation for second round breast cancer screening: a randomised controlled trial. fournal of Medical Screening 1994;1:24548.

8 Taylor V, Taplin S, Urban N, White E, Peacock S. Repeat mammography use among women ages 50-70. Cancer Epidemiology, Biomarkers and Prevention 1995;4:409-13.

9 UK Trial of Early Detection of Breast Cancer Group. First results on mortality reduction in the UK trial of early detection of breast cancer. Lancet 1988;i:411-16.

10 Peeters PHM, Verbeek ALM, Hendriks JHCL, van Bon MJH. Screening for breast cancer in Nijmegen. Report of 6 screening rounds, 1975-1986. Br f Cancer 1989;43: 626-30.

11 Schofield PE, Cockburn J, Hill DJ, Reading D. Encouraging ttendance at a mammography screening program: determinants of response to different recruitment strategies. fournal of Medical Screening 1994;1:144-49.

12 Cockburn J, Murphy B, Schofield P, Hill D, Borland R. Development of a strategy to encourage attendance for screening mammography. Health Education and Research 1991;6:279-90.

13 Mischel W. Personality and assessment. New York: Wiley, 1968

14 Turnbull D, Adelson P, Irwig L. Evaluating the impact of a promotional campaign for screening mammography: women's knowledge and sources of awareness. Aust Public Health 1992;16:72-8. 